\title{
MANIFOLDS WITH NO PERIODIC HOMEOMORPHISMS
}

\author{
BY
}

\author{
EDWARD M. BLOOMBERG(1)
}

\begin{abstract}
An analysis of the ends of the universal cover of the connected sum of two aspherical manifolds leads to the construction of a class of closed manifolds with no nontrivial periodic homeomorphisms.
\end{abstract}

This paper exhibits a class of closed four dimensional manifolds on which no compact Lie group can act effectively. No assumption of smoothness is made. I have been told [5] that before any such manifold was born, it had been named "The Ugly Manifold". But the one in this paper is a simple manifold to construct. The building blocks are the manifolds, $L(k)$, constructed in [1]. These are four dimensional $K(\pi, 1)$ 's with the property that any finite group that acts on one of them must act freely. Then

MAIN THEOREM. If $k$ and $k^{\prime}$ are distinct primes, then $L=L(k) \#$ $L(k$ ') has no compact Lie group acting effectively on it ("\#" denotes connected sum).

Here is an outline of the proof:

(1) $\tau$, the universal cover of $L$, is shown to be embeddable as a dense subset of $S^{4}$, the four sphere, in such a way that $S^{4}-\tilde{L}$ can be identified with the ends of $\tilde{L}$. That is, $S^{4}$ is the Freudenthal end point compactification of $\tilde{L}$.

(2) The outer automorphism group of $\pi_{1}(L)$ is calculated and shown to be torsion free.

(3) This allows us to conclude that if $h$ were a periodic homeomorphism of $L$, then $h$ would lift to a periodic homeomorphism, $\tilde{h}$, of $\widetilde{L}$ and hence $\widetilde{h}$ extends to a periodic homeomorphism, $H$, of $S^{4}$.

Received by the editors July 24, 1973.

AMS (MOS) subject classifications (1970). Primary 57E25; Secondary 20E30.

Key words and phrases. Aspherical manifolds, Freudenthal end point compactification, connected sums, free products, outer automorphism group.

(1) This paper contains the results of the author's dissertation submitted in partial fulfillment of the requirements for the degree of Doctor of Philosophy at the University of Michigan. 
Furthermore, $H$ commutes with the action of $\pi_{1}(L)$ that is induced on $S^{4}$.

(4) The action of $\pi_{1}(L)$ on the ends of $\tilde{L}$ is completely determined. Since $H$ commutes with this action, this allows us to conclude that $H$ leaves the ends of $\tilde{L}$ pointwise fixed.

(5) Since the $Z_{p}$-cohomology of the fixed point set of $H$ and therefore of $\tilde{h}$ can be computed, we consider an appropriate spectral sequence relating the fixed point set of $\widetilde{h}$ with that of $h$. A contradiction is obtained using elementary results about the cohomology of groups.

At the time that this paper was written, Conner and Raymond had constructed the manifolds $L(k)$ on which any finite group which acted effectively acted freely. They have now extended their results, using techniques different from those in this paper, to obtain manifolds with no action in dimensions $n(n+1) / 2+1,3 \leqslant n \leqslant 8$.

In conclusion, a word about motivation. (I wish that all authors would include such a paragraph in their works.) Conner and Raymond obtained their examples in [1] by examining $K(\pi, 1)$ 's which were finite dimensional manifolds. For any $Z_{p}$ action on a $K(\pi, 1)$, they obtained explicit formulae for the $Z_{p}$ extension of $\pi$ that acts on $\overparen{K(\pi, 1)}$. They then performed their calculations on $\overparen{K(\pi ; 1)}$, which was homeomorphic to $R^{n}$, Euclidean $n$-space. In the example at hand, it is evident that $L$ is a candidate for a manifold with no symmetry. But it was unclear how to handle it, since $L$ is, of course, not a $K(\pi, 1)$. Although the universal cover of $L$ is unmanageable, its Freudenthal end point compactification is $S^{4}$. So instead of looking at universal covers (that is, $R^{n}$ ) à la Conner and Raymond, we look at the maximal zero dimensional compactification of the universal cover (that is, $S^{n}$ ).

I. Out $\left(L(k) * L\left(k^{\prime}\right)\right)$ is torsion free.

1.1. This section fixes notation and provides the algebraic background needed for the rest of the paper. The general reference for free products is [3]. and $A^{\prime}$.

If $A$ and $A^{\prime}$ are groups, $A * A^{\prime}$ denotes the free product of $A$

Definition. Out $A$ is the quotient of the group of automorphisms of $A$ by the group of inner automorphisms of $A$.

Definition. A group $A$ is called prime if it is not isomorphic to a nontrivial free product or an infinite cyclic group.

THEOREM 1.1.1. If $A$ and $A^{\prime}$ are prime groups, then:

(a) If $A$ and $A^{\prime}$ are not isomorphic Out $\left(A * A^{\prime}\right)=$ Aut $A \oplus$ Aut $A^{\prime}$. 
(b) If $A$ and $A^{\prime}$ are isomorphic Out $\left(A * A^{\prime}\right)=($ Aut $A \oplus$ Aut $A^{\prime}$ ) $\circ Z_{2}$, the semidirect product of (Aut $A \oplus$ Aut $A^{\prime}$ ) and $Z_{2}$, where $Z_{2}$ acts on Aut $A \oplus$ Aut $A^{\prime}$ by switching factors.

Proof. (a) Let $\Gamma \in$ Aut $\left(A * A^{\prime}\right)$. Then $\Gamma(A)$ and $\Gamma\left(A^{\prime}\right)$ are subgroups of $A * A^{\prime}$. By the Kurosh subgroup theorem [3, p. 243]

$$
\Gamma(A) \subset \gamma F \gamma^{-1}, \quad \Gamma\left(A^{\prime}\right) \subset \gamma^{\prime} F^{\prime} \gamma^{\prime-1},
$$

where $F$ and $F^{\prime}$ are either $A$ or $A^{\prime}$, and $\gamma, \gamma^{\prime}$ are in $A * A^{\prime}$.

Lemma. $\Gamma(A)=\gamma A \gamma^{-1}, \Gamma\left(A^{\prime}\right)=\gamma^{\prime} A^{\prime} \gamma^{\prime-1}$.

Proof. Suppose $\Gamma(A)$ were a proper subgroup of $\gamma F \gamma^{-1}$. Then $A * A^{\prime}$ would be generated by $F_{1}$ and $\left(\gamma^{-1} \gamma^{\prime}\right) F^{\prime}\left(\gamma^{-1} \gamma^{\prime}\right)^{-1}$ where $F_{1}$ is a proper subgroup of $F$. It is elementary to see that the only possibility for $\gamma^{-1} \gamma^{\prime}=a a^{\prime}$ where $a \in F$ and $a^{\prime} \in F^{\prime}$. But then the group generated by $F_{1}$ and $\left(a a^{\prime}\right) F^{\prime}\left(a a^{\prime}\right)^{-1}$ is also generated by $a^{-1} F_{1} a$ and $a^{\prime} F^{\prime} a^{\prime-1}=F^{\prime}$. But $a^{-1} F_{1} a$ is a proper subgroup of $F$. Certainly $F=F^{\prime}$ is impossible. But also, if $A_{1}$ is a proper subgroup of $A$, then $A_{1} * A^{\prime}$ is a proper subgroup of $A * A^{\prime}$. So $F_{1}$ could not be a proper subgroup of $F$.

Therefore $\Gamma(A)=\gamma F \gamma^{-1}$. Since $A$ and $A^{\prime}$ are not isomorphic, $F=A$.

Similarly $\Gamma\left(A^{\prime}\right)=\gamma^{\prime} A^{\prime} \gamma^{\prime-1}$.

Let $\mu_{\gamma} \in \operatorname{Aut}\left(A * A^{\prime}\right)$ be conjugation by $\gamma$, where $\gamma \in A * A^{\prime}$. Then

$$
\begin{aligned}
& \mu_{\gamma^{-1}} \circ \Gamma: A \rightarrow A, \\
& \mu_{\gamma^{-1}} \circ \Gamma: A^{\prime} \rightarrow\left(\gamma^{-1} \gamma^{\prime}\right) A^{\prime}\left(\gamma^{-1} \gamma^{\prime}\right)^{-1} .
\end{aligned}
$$

Again, $\gamma^{-1} \gamma^{\prime}=a a^{\prime}$ for some $a \in A, a^{\prime} \in A^{\prime}$. But then

$$
\alpha=\mu_{a^{-1} \gamma^{-1}} \circ \Gamma: A \rightarrow A, \quad \alpha^{\prime}=\mu_{a^{-1} \gamma^{-1}} \circ \Gamma: A^{\prime} \rightarrow A^{\prime} .
$$

For any $\alpha \in$ Aut $A, \alpha^{\prime} \in$ Aut $A^{\prime}$, let $\left[\alpha, \alpha^{\prime}\right] \in \operatorname{Aut}\left(A * A^{\prime}\right)$ be the automorphism determined by $\left.\left[\alpha, \alpha^{\prime}\right]\right|_{A}=\alpha,\left.\left[\alpha, \alpha^{\prime}\right]\right|_{A^{\prime}}=\alpha^{\prime}$. We have seen so far that for each $\Gamma \in \operatorname{Aut}\left(A * A^{\prime}\right)$ there is an $\eta \in A * A^{\prime}, \alpha \in$ Aut $A, \alpha^{\prime} \in$ Aut $A^{\prime}$ such that $\Gamma=\mu_{\eta} \circ\left[\alpha, \alpha^{\prime}\right]$.

IEMMA. The above representation is unique.

Proof. Suppose $\mu_{\eta_{1}} \circ\left[\alpha_{1}, \alpha_{1}^{\prime}\right]=\mu_{\eta_{2}} \circ\left[\alpha_{2}, \alpha_{2}^{\prime}\right]$, then $\left[\alpha_{1}, \alpha_{1}^{\prime}\right]=$ $\mu_{\eta_{1}{ }^{1} \eta_{2}} \circ\left[\alpha_{2}, \alpha_{2}^{\prime}\right]$. So the image of $A$ under the automorphism $\left[\alpha_{1}, \alpha_{1}^{\prime}\right]$ is the same as its image under $\mu_{\eta_{1}{ }^{1} \eta_{2}} \circ\left[\alpha_{2}, \alpha_{2}^{\prime}\right]$. That is

$$
A=\left(\eta_{1}^{-1} \eta_{2}\right) A\left(\eta_{1}^{-1} \eta_{2}\right)^{-1} \text {. }
$$


By [3, p. 187], $\eta_{1}^{-1} \eta_{2} \in A$. Similarly, $\eta_{1}^{-1} \eta_{2} \in A^{\prime}$. So $\eta_{1}^{-1} \eta_{2}=1$. That is $\eta_{1}=\eta_{2}$. But then

$$
\left[\alpha_{1}, \alpha_{1}^{\prime}\right]=\left[\alpha_{2}, \alpha_{2}^{\prime}\right] \Rightarrow \underset{\alpha_{1}}{\left[\alpha_{1}\right.},\left.\underset{\alpha_{1}^{\prime}}{\alpha_{1}^{\prime}}\right|_{A}=\left[\left.{\underset{\alpha}{2}}_{\alpha_{2}}^{\left.\alpha_{2}^{\prime}\right]}\right|_{A} .\right.
$$

Similarly $\alpha_{1}^{\prime}=\alpha_{2}^{\prime}$.

Therefore, any automorphism of $A * A^{\prime}$ is uniquely represented by $\mu_{\eta} \circ\left[\alpha, \alpha^{\prime}\right]$ for $\eta \in A * A^{\prime}, \alpha \in$ Aut $A, \alpha^{\prime} \in$ Aut $A^{\prime}$.

Define a function $\Phi:$ Aut $\left(A * A^{\prime}\right) \rightarrow$ Aut $A \oplus$ Aut $A^{\prime}$ by $\Phi\left(\mu_{\eta} \circ\left[\alpha, \alpha^{\prime}\right]\right)=\left(\alpha, \alpha^{\prime}\right)$.

It is straightforward to verify that $\Phi$ is an epimorphism with kernel, the group of inner automorphisms of $A * A^{\prime}$. Hence the result.

(b) The proof of part (b) is similar, but since it will not be used in this paper it is omitted.

COROLlaRY 1.1.2. If $A$ and $A^{\prime}$ are nonisomorphic prime groups and Aut $A$ and Aut $A^{\prime}$ are torsion free, then $\operatorname{Out}\left(A * A^{\prime}\right)$ is torsion free.

II. The Freudenthal end point compactification of connected sums.

2.1. This section provides a convenient indexing of the universal cover of the connected sum of two manifolds of dimension greater than two.

Suppose $M$ and $M^{\prime}$ are manifolds of dimension greater than two. Let $M_{1}$ (resp. $M_{1}^{\prime}$ ) denote $M$ - open disk (resp. $M^{\prime}$ - open disk). Denote by $A$ (resp. $A^{\prime}$ ) the fundamental group of $M$ (resp. $M^{\prime}$ ); then $A * A^{\prime}$ is isomorphic to the fundamental group of $M \# M^{\prime}$. Let $A * A^{\prime} / A$ be the set of left cosets of $A$. Let [ ]:A*A' $\rightarrow A * A^{\prime} / A, \gamma \rightarrow[\gamma]$ be the projection map.

Similarly for [ ]': $A * A^{\prime} \rightarrow A * A^{\prime} / A^{\prime}$. For each $[\gamma] \in A * A^{\prime} / A$, let $\widetilde{M}_{[\gamma]}$ denote a copy of $M_{1}$. For each $[\gamma]^{\prime} \in A * A^{\prime} / A^{\prime}$, let $\widetilde{M}_{[\gamma]}^{\prime}$, denote a copy of $M_{1}^{\prime}$.

Choose a sphere in $\partial \widetilde{M}_{1}$ and call it $S_{1}$. For each $a \in A$, let $S_{a}=a\left(S_{1}\right)$, where $A$ acts on $\widetilde{M}_{1}$ on the left as covering transformations. Since $\widetilde{M}_{[\gamma]}$ is homeomorphic to $\widetilde{M}_{1}$, let $S_{\gamma a} \in \widetilde{M}_{[\gamma]}$ correspond to $S_{a}$, where $\gamma \in A * A^{\prime}$ has reduced form ending with an element in $A^{\prime}$. Similarly, index $S_{\gamma}^{\prime} \in \widetilde{M}_{[\gamma]}^{\prime}$.

Choose a homeomorphism $\phi_{1}: S_{1} \rightarrow S_{1}^{\prime}$. Then there is a unique homeomorphism $\phi_{\gamma}: S_{\gamma} \rightarrow S_{\gamma}^{\prime}, \gamma \in A * A^{\prime}$ compatible with the identifications of $\widetilde{M}_{\gamma}, \widetilde{M}_{\gamma}^{\prime}$ and $\phi_{1}$.

PROPOSITION 2.1.1. $X=\widetilde{M}_{[\gamma]} \cup_{\phi_{\gamma ; \gamma} \in A * A}, \widetilde{M}_{[\gamma]}^{\prime}$, is the universal cover of $M_{1} \cup_{\phi_{1}} M_{1}^{\prime}=M \# M^{\prime}$. 
Proof. The projection map from $X$ to $M \# M^{\prime}$ is the obvious one.

To describe the action of $A * A^{\prime}$ on $X$ : For each $[\gamma] \in A * A^{\prime} / A$, there is a unique $\gamma_{0} \in A * A^{\prime}$ such that $\left[\gamma_{0}\right]=[\gamma]$ and the (syllable) length of $\gamma_{0}$ is minimal. Denote the homeomorphism from $\widetilde{M}_{[\gamma]}$ to $\widetilde{M}_{1}$ by $H_{\gamma_{0}}$. For $a \in A$, define $H_{\gamma_{0} a}$ to be the composite

$$
\widetilde{M}_{[\gamma]} \stackrel{{ }_{\gamma_{0}}}{\longrightarrow} \widetilde{M}_{1} \stackrel{a^{-1}}{\longrightarrow} \widetilde{M}_{1}
$$

Then $H_{\gamma}$ is defined for all $\gamma \in A * A^{\prime}$. Similarly for $H_{\gamma}^{\prime}: \widetilde{M}_{[\gamma]^{\prime}}^{\prime} \rightarrow \widetilde{M}_{1}^{\prime}$. Then the action of $A * A^{\prime}$ on $X$ is defined by: if $\alpha \in A * A^{\prime}$, then $\nu_{\alpha}: X \rightarrow X$ is given by

$$
\left.\nu_{\alpha}\right|_{\tilde{M}_{[\gamma]}}=H_{\alpha[\gamma]}^{-1} \circ H_{[\gamma]},\left.\quad \nu_{\alpha}\right|_{\tilde{M}_{[\gamma]}^{\prime}}=H_{\alpha[\gamma]^{\prime}}^{\prime-1} \circ H_{[\gamma]^{\prime}}^{\prime}
$$

It is a straightforward calculation to verify that $X$ is the universal cover of $M \# M^{\prime}$ and the $\nu_{\alpha}$ give the left action of $A * A^{\prime}$ on $X$ as covering transformations.

2.2. Assume now that $\widetilde{M}$ and $\widetilde{M}^{\prime}$ are Euclidean $n$-space, $n>2$. This section provides an embedding of $\widetilde{M \# M^{\prime}}$ in $S^{n}$ in such a way that $S^{n}$ can be identified with the Freudenthal end point compactification of $\overparen{M \# M^{\prime}}$.

Since $\widetilde{M}$ is homeomorphic to $R^{n}$ and $S^{n}$ is the one point compactification of $R^{n}$, there is an embedding of $\widetilde{M}_{1}$ in $S^{n}$. Similarly there is an embedding of $\widetilde{M}_{1}^{\prime}$ in $S^{n}$. Recall that $\partial \widetilde{M}_{1}=\bigcup_{a \in A} S_{a}^{n-1}$. Each $S_{a}^{n-1}$ bounds a disk in $S^{n}-\widetilde{M}_{1}$. Call the disk bounded by $S_{a}^{n-1}, D_{a}^{n}$. Embed $\widetilde{M}_{1}^{\prime}$ in $D_{1}$ so that the embedding of $S_{1}^{\prime} \subset \widetilde{M}_{1}^{\prime}$ in $S^{n}$ is compatible with the embedding of $S_{1} \subset \widetilde{M}_{1}$ in $S^{n}$. Then

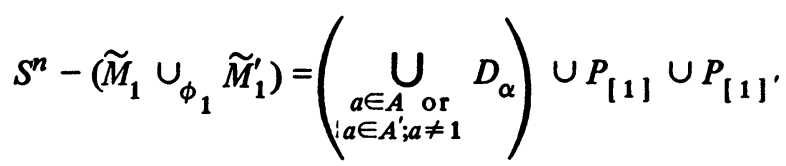

where $P_{[1]}$ and $P_{[1]}$, are points.

Suppose we have embedded $\left(\cup \widetilde{M}_{[\gamma]}\right) \cup\left(\cup \widetilde{M}_{[\gamma]^{\prime}}^{\prime}\right)$ in $S^{n}$ where the union is taken over all $[\gamma]$ with the length of $\gamma_{0},\left|\gamma_{0}\right|$, less than or equal to $k$. (See $\S 2.1$ for the definition of $\gamma_{0}$.) Then

$$
\begin{aligned}
S^{n}-\left(U \widetilde{M}_{[\gamma]}\right) \cup\left(U \widetilde{M}_{[\gamma]^{\prime}}^{\prime}\right)= & \left(\bigcup_{\left|\gamma_{0}\right|=k+1} D_{[\gamma]}\right) \cup\left(\bigcup_{\left|\gamma_{0}\right|=k+1} D_{[\gamma]^{\prime}}\right) \\
& \cup\left(\bigcup_{\left|\gamma_{0}\right|<k} P_{[\gamma]}\right) \cup\left(\bigcup_{\left|\gamma_{0}\right|<k} P_{[\gamma]^{\prime}}\right) .
\end{aligned}
$$


There is, then, an obvious embedding of $\widetilde{M}_{[\gamma]}$ and $\widetilde{M}_{[\gamma]^{\prime}}^{\prime}$ in $D_{[\gamma]}$ where $\left|\gamma_{0}\right|=k+1$, which is compatible with the identifications in $X$. If the embeddings of $\widetilde{M}_{[\gamma]}$ and $\widetilde{M}_{[\gamma]^{\prime}}^{\prime}$ in $S^{n}$ are such that the disks formed at the $k$ th stage have diameter $\leqslant \epsilon_{k}$ where $\lim _{k \rightarrow \infty} \epsilon_{k}=0$, then $S^{n}-X=K$ is a cantor set. Further $K=P \cup D$ where

$$
P=\left(\underset{[\gamma] \in A^{*} A^{\prime} / A}{ } P_{[\gamma]}\right) \cup\left(\underset{[\gamma]^{\prime} \in A^{*} A^{\prime} / A^{\prime}}{\bigcup_{[\gamma]^{\prime}}}\right)
$$

and

$$
D=\bigcup_{\left\{\gamma_{i}\right\}_{i=1}^{\infty}}\left(\bigcup_{i=1}^{\infty} D_{\gamma_{i}}\right)
$$

where the union in the latter set is taken over all sequences $\left\{\gamma_{i}\right\}_{i=1}^{\infty}$ where $\gamma_{i}$ $\in A * A^{\prime},\left|\gamma_{i}\right|=i$ and $\gamma_{i+1}=\gamma_{i} a$ or $\gamma_{i+1}=\gamma_{i} a^{\prime}, a \in A, a^{\prime} \in A^{\prime}$-whichever one is in reduced form. Call such a sequence $\left\{\gamma_{i}\right\}$ admissible. Notice that $P$ is dense in $K$. We shall consider $P_{[1]^{\prime}}$ as the base point of $K$.

Finally notice that the action of $A * A^{\prime}$ on $\overparen{M \# M^{\prime}}$ extends naturally to an action on $K$ by the formulae: if $\alpha \in A * A^{\prime}$, then

$$
\begin{array}{ll}
\nu_{\alpha}\left(P_{[\gamma]}\right)=P_{[\alpha \gamma]}, & \nu_{\alpha}\left(P_{[\gamma]^{\prime}}\right)=P_{[\alpha \gamma]^{\prime}}, \\
\nu_{\alpha}\left(\bigcap_{i} D_{\gamma_{i}}\right)=\bigcap_{i>k} D_{\alpha \gamma_{i}} & \begin{array}{l}
\text { where } k \text { is such that }\left|\alpha \gamma_{k}\right| \text { is the minimal length } \\
\text { of any }
\end{array}
\end{array}
$$

The following results will be needed later.

Proposition 2.2.1. Let $\alpha \in A * A^{\prime}$ act on $K$ as above. Then the fixed point set of $\alpha$ restricted to $K$ is

(i) if $\alpha=\beta a \beta^{-1}$ for some $1 \neq a \in A$, then the fixed point set is $P_{[\beta]}$;

(ii) if $\alpha=\beta a^{\prime} \beta^{-1}$ for some $1 \neq a^{\prime} \in A^{\prime}$, then the fixed point set is $P_{[\beta]^{\prime}}$;

(iii) if $\alpha=\beta \omega \beta^{-1}$ for some $\omega \in A * A^{\prime}$, where $|\omega|>1$ and $|\beta|$ is maximal for such a representation then the fixed point set is $\bigcap_{i} D_{\gamma_{i}}$ where $\left\{\gamma_{i}\right\}$ is the admissible sequence containing the words $\gamma \omega^{i}$ for all $i$.

Proof. Suppose that $\alpha \in A * A^{\prime}$ satisfies $\alpha\left(P_{[\gamma]}\right)=P_{[\gamma]}$, then $P_{[\alpha \gamma]}=\alpha P_{[\gamma]}=P_{[\gamma]}$ implies there exists $a \in A$ such that $\alpha \gamma=\gamma a$; that is $\alpha=\gamma a \gamma^{-1}$. Similarly, $\alpha\left(P_{[\gamma]^{\prime}}\right)=P_{[\gamma]^{\prime}}$ implies there exists an $a^{\prime} \in A^{\prime}$ such that $\alpha=\gamma a^{\prime} \gamma^{-1}$. So the only elements $\alpha \in A * A^{\prime}$ that fix an element of $P$ are of the form $\gamma x \gamma^{-1}$ for $x \in A$ or $x \in A^{\prime}$. Suppose $\alpha\left(\bigcap_{i} D_{\gamma_{i}}\right)=\bigcap_{i} D_{\gamma_{i}}$ for $\left\{\gamma_{i}\right\}$ an admissible sequence, and $\alpha=\gamma x \gamma^{-1}, x \in A$ or $x \in A^{\prime}$. Then, since $\left|\alpha^{2}\right|=|\alpha|$, we would have for some $i$ large enough, $\alpha^{2} \gamma_{i}=\alpha \gamma_{i}$. This 
implies that $\alpha=1$. This proves parts (i) and (ii). Suppose $\alpha=\beta \omega \beta^{-1}$ where $|\omega|>1$ and $|\beta|$ is maximal. Then, by the above, $\alpha$ does not fix any point of $P$. If $\alpha\left(\bigcap_{i} D_{\gamma_{i}}\right)=\bigcap_{i} D_{\gamma_{i}}$, let $n$ be an integer such that $\left\{\alpha \gamma_{i}\right\}_{i=n}^{\infty}$ is an admissible sequence. Then the sequence $\left\{\alpha \gamma_{i}\right\}_{i=n}^{\infty}$ contains the sequence $\left\{\alpha^{k} \gamma_{n}\right\}_{k=1}^{\infty}$. Since $|\omega|>1$, this implies that $\left\{\alpha \gamma_{i}\right\}_{i=n}^{\infty}$ contains the sequence $\left\{\gamma \omega^{i}\right\}_{i=1}^{\infty}$.

Corollary 2.2.2. $\widetilde{H}^{0}(K ; Z)$ is isomorphic to $Z\left(A * A^{\prime}\right)$ as $Z\left(A * A^{\prime}\right)$ modules, where $\widetilde{H}^{*}$ denotes reduced Čech cohomology with $Z\left(A * A^{\prime}\right)$ module structure induced by the action of $A * A^{\prime}$ on $K$, and $Z\left(A * A^{\prime}\right)$ is a $Z\left(A * A^{\prime}\right)$ module by left multiplication.

Proof. We interpret $\widetilde{H}^{0}(X, Z)$ as the $Z$-module of locally constant functions from $X$ to $Z$ whose value on the base point of $X$ is 0 . The $Z\left(A * A^{\prime}\right)$ structure is given by: if $\alpha \in A * A^{\prime}$ and $f: X \rightarrow Z$ is locally constant, then $\alpha f(x)=f(\alpha x)$.

Notation. If $1 \neq \alpha \in A * A^{\prime}$ let $(\alpha) \in A * A^{\prime}$ be the word obtained from $\alpha$ by deleting the last syllable in the reduced form of $\alpha$. (If $\alpha \in A$ or $A^{\prime}$, define $(\alpha)=1$.)

Let $f \in \widetilde{H}^{0}(K, Z)$ be a locally constant function from $K$ to $Z$. Define

$$
\chi: \widetilde{H}^{0}(K, Z) \rightarrow \operatorname{Hom}_{Z}\left(Z\left(A * A^{\prime}\right), Z\right)
$$

by $\chi(f)(1)=f\left(P_{1}\right), \chi(f)(\alpha)=f\left(P_{\alpha}\right)-f\left(P_{(\alpha)}\right)$ for $\alpha \neq 1$. (I am abusing notation here. I should write $\chi(f)(\alpha)=f\left(P_{[\alpha]}\right)-f\left(P_{[(\alpha)]^{\prime}}\right)$ if $\alpha$ ends with an element of $A^{\prime},=f\left(P_{[\alpha]^{\prime}}\right)-f\left(P_{[(\alpha)]}\right)$ if $\alpha$ ends with an element of $A$.)

Lemma. Image $\chi \subset \operatorname{Hom}_{Z}^{c}\left(Z\left(A * A^{\prime}\right), Z\right)$-the submodule of functions $\phi: Z\left(A * A^{\prime}\right) \rightarrow Z$ such that $\phi(\alpha)=0$ for all but a finite number of $\alpha \in A * A^{\prime}$.

Proof. If $P_{\gamma_{1}}, P_{\gamma_{2}}, \cdots$ is an infinite sequence of distinct points in $P$ such that $\chi(f)\left(\gamma_{i}\right) \neq 0$ for each $i$, then some subsequence of $P_{\gamma}$ 's converges to a point $k \in K$. To avoid cumbersome notation call this new sequence $P_{\gamma_{1}}, P_{\gamma_{2}}, \cdots$. Then $\left\{P_{\gamma_{i}}\right\}$ lies eventually in any disk $D_{\gamma}, k \in D_{\gamma}$. Then $\left\{P_{\gamma_{i}}\right\}$ and $\left\{P_{\left(\gamma_{i}\right)}\right\}$ lie eventually in any disk $D_{\gamma}$ containing $k$.

Since $f$ is locally constant, we may choose $D_{\gamma}$ small enough so that $f l_{D_{\gamma}}$ is constant. Then $f\left(P_{\gamma_{i}}\right)=f\left(P_{\left(\gamma_{i}\right)}\right)$ for $i$ large enough. So $\chi(f)\left(\gamma_{i}\right)=$ $f\left(P_{(\gamma)}\right)-f\left(P_{\left(\gamma_{i}\right)}\right)=0$ for $i$ large enough, contradicting the choice of $\gamma_{i}$.

Claim. $\chi$ is an isomorphism of $Z(\pi)$ modules:

(a) $\chi$ is one to one: If $f \neq g, f, g \in \widetilde{H}^{0}(K, Z)$, then $\left.f\right|_{P} \neq\left. g\right|_{P}$ since $P$ is dense in $K$. So there exists a $P_{\gamma} \in P,|\gamma|$ minimal such that $f\left(P_{\gamma}\right) \neq g\left(P_{\gamma}\right)$. If $\gamma=1$, then $\chi(f)(1)=f\left(P_{1}\right) \neq g\left(P_{1}\right)=\chi(g)(1)$. If $|\gamma| \geqslant 1$, then, $f\left(P_{(\gamma)}\right)=$ $g\left(P_{(\gamma)}\right)$ from the choice of $\gamma$. So 


$$
\chi(f)(\gamma)=f\left(P_{\gamma}\right)-f\left(P_{(\gamma)}\right)=f\left(P_{\gamma}\right)-g\left(P_{(\gamma)}\right) \neq g\left(P_{\gamma}\right)-g\left(P_{(\gamma)}\right)=\chi(g)(\gamma) .
$$

That is $\chi(f) \neq \chi(g)$.

(b) $\chi$ is onto.

If $F \in \operatorname{Hom}_{Z}^{c}\left(Z\left(A * A^{\prime}\right), Z\right)$, define, for $P_{\gamma} \in P, f\left(P_{\gamma}\right)=\Sigma_{\alpha} F(\alpha)$ where the sum is taken over all $\alpha$ obtained from $\gamma$ by successively deleting the last $n$ terms of $\gamma$ where $0 \leqslant n \leqslant|\gamma|$. It is elementary to show that $f$ has an extension to $K$ that is a locally constant map from $K$ to $Z$. Then

$$
\chi(f)(\gamma)=f\left(P_{\gamma}\right)-f\left(P_{(\gamma)}\right)=F(\gamma):
$$

that is $\chi(f)=F$.

(c) $\chi$ is a $Z\left(A * A^{\prime}\right)$ homomorphism. The proof is straightforward. This completes the proof since of course $\operatorname{Hom}_{Z}^{c}\left(Z\left(A * A^{\prime}\right), Z\right) \approx Z\left(A * A^{\prime}\right)$ as $Z\left(A * A^{\prime}\right)$ modules.

2.3. This section completes the demonstration that if $\widetilde{M}=R^{n}$ and $\widetilde{M}^{\prime}=$ $R^{n}$, then the Freudenthal end point compactification of $\widetilde{M \# M^{\prime}}$ is $S^{n}$. The general reference for this is [4].

In [4], Raymond constructs a natural topology on the ends $E(X)$ of a space $X$ and characterizes the natural topology on $X \cup E(X)=F(X)$, the Freudenthal end point compactification of $X$. Using this result [4, p. 949] it is easy to see that $S^{n}=X \cup K$ is the Freudenthal end point compactification of $X$, where $X$ and $K$ are as in $\$ 2.2$.

The primary feature of the end point compactification that we will use is:

Proposition 2.3.1 (RAYMOND). Let $X$ be a connected, locally connected, locally compact Hausdorff space. Let $h: X \rightarrow X$ be a homeomorphism. Then $h$ has a unique extension $H: F(X) \rightarrow F(X)$.

Proof (using the notation of [4]). Define $H$ as follows:

If $V_{\alpha}$ is an open set with compact closure, then so is $h\left(V_{\alpha}\right)=V_{\alpha^{\prime}} \cdot h$ takes the unbounded components of $X-\bar{V}_{\alpha}$ onto the unbounded components of $X-\bar{V}_{\alpha^{\prime}}$. Furthermore, we have the commutative diagram:

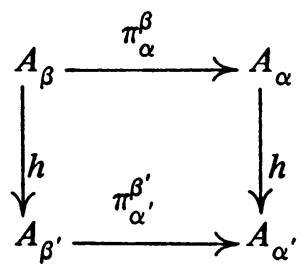

We thus have a map $h^{\prime}: B \rightarrow B$ which is one to one, onto and continuous, since $\left.h\right|_{A \alpha}$ is. Since $B$ is compact, $h^{\prime}$ is a homeomorphism. 
Define $H: F(X) \rightarrow F(X)$ by $\left.H\right|_{X}=h,\left.H\right|_{B}=h^{\prime}, H$ is clearly the unique extension of $h$.

\section{The example.}

3.1. In [1], Conner and Raymond constructed closed four dimensional manifolds, $L(k), k \geqslant 2$, with the property that every finite group which acts on an $L(k)$ must act freely. These manifolds are $K(\pi, 1)$ 's-Eilenberg-Mac Lane spaces of type $(\pi, 1)$. Set $\pi_{1} L(k)=L(k)$. The properties of these manifolds that we will use are summarized in

PROPOSITION 3.1.1 (SEE' [1]). (1) $\widetilde{L(k)}=R^{4}$. (2) Aut $L(k)$ is torsion free. (3) $L(k)$ and $L\left(k^{\prime}\right)$ are not isomorphic for $k \neq k^{\prime}$.

Main Theorem. If $h: L(k) \# L\left(k^{\prime}\right) \rightarrow L(k) \# L\left(k^{\prime}\right), k \neq k^{\prime}$ is a periodic homeomorphism, then $h=\mathrm{id}$. Hence no compact Lie group acts effectively on $L(k) \# L\left(k^{\prime}\right)$.

Proof. Suppose $h$ is a nontrivial periodic homeomorphism of $L(k) \#$ $L\left(k^{\prime}\right)$ of period $p$, a prime. Then $h$ lifts to a homeomorphism $\tilde{h}$ of $X=$ $L(k) \# L\left(k^{\prime}\right)$. The discussion in II established that $F(X)=S^{4}$; i.e. $S^{4}$ is the Freudenthal end point compactification of $X$. So by Proposition 2.3.1, $\widetilde{h}$ extends uniquely to a homeomorphism $H$ of $S^{4}$. ( $H$ is a homeomorphism, since $\tilde{h}^{-1}$ has a unique extension, $H^{-1}$ to $S^{4}$.) That is we have the following commutative diagram:

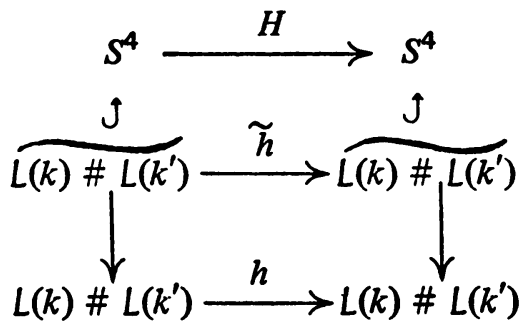

Of course, neither $\widetilde{h}$ nor $H$ need be, a fortiori, periodic. However $\widetilde{h}$ does provide a $Z_{p}$ extension, $\Lambda$, of $L(k) * L\left(k^{\prime}\right)$, that does act on $S^{4}$; namely $0 \rightarrow L(k) * L\left(k^{\prime}\right) \rightarrow \Lambda \rightarrow Z_{p} \rightarrow 1$ with data

$$
\begin{aligned}
& \Phi: Z_{p} \rightarrow \operatorname{Out}\left(L(k) * L\left(k^{\prime}\right)\right) \text {, a homomorphism, } \\
& f: Z_{p} \times Z_{p} \rightarrow L(k) * L\left(k^{\prime}\right) \text {, a function, }
\end{aligned}
$$

satisfying certain compatibility conditions (see [1] and [2]). $\Phi$ is determined by:

$$
\begin{array}{cc}
\Phi(1)=h_{*}: \pi_{1}\left(L(k) \# L\left(k^{\prime}\right), x\right) \rightarrow \pi_{1}\left(L(k) \# L\left(k^{\prime}\right), h(x)\right) \\
\text { ॥ } & \text { \# } \\
L(k) * L\left(k^{\prime}\right) & L(k) * L\left(k^{\prime}\right)
\end{array}
$$


Notice that since $h$ does not have, in general, a fixed point, $h_{*}$ is only determined up to an inner automorphism of $L(k) * L\left(k^{\prime}\right)$.

Congruence classes of extensions are classified by

$$
H_{\Phi}^{2}\left(Z_{p}, \operatorname{Center}\left(L(k) * L\left(k^{\prime}\right)\right)\right)=0,
$$

since center $(A * B)=0$ for any nontrivial $A$ and $B$.

Lemma. $\Phi: Z_{p} \rightarrow \operatorname{Out}\left(L(k) * L\left(k^{\prime}\right)\right)$ is the zero map.

Proof. Certainly $L(k)$ and $L\left(k^{\prime}\right)$ are prime groups (see $\S 1.1$ for definition). Then Proposition 3.1.1 and Corollary 1.1.2 show that $\operatorname{Out}\left(L(k) * L\left(k^{\prime}\right)\right)$ is torsion free.

Therefore $\Lambda$ is the unique $Z_{p}$ extension of $L(k) * L\left(k^{\prime}\right)$ with data $\Phi=0$. Therefore $\Lambda \approx\left(L(k) * L\left(k^{\prime}\right)\right) \oplus Z_{p}$, i.e. $Z_{p}$ acts on $S^{4}$. Hence

PROPOSITION 3.1.2. There exists a lift $\tilde{h}$ of $h$, and therefore an $H$ that is periodic with period $p$ and that commutes with the action of $L(k) * L\left(k^{\prime}\right)$.

Using the notation of $\S 2.2: S^{4}=F(X)=X \cup K$ and $K=P \cup D$.

Proposition 3.1.3. $\left.H\right|_{K}=$ identity.

Proof. We only use the fact that $H$ commutes with the action of $L(k) * L\left(k^{\prime}\right)$ on $K$.

Consider $P_{[1]} \in P$.

Case 1. $H\left(P_{[1]}\right) \in P$ and $H\left(P_{[1]^{\prime}}\right) \in P$.

(a) Suppose $H\left(P_{[1]}\right)=P_{[\alpha]}$ and $H\left(P_{[1]^{\prime}}\right)=P_{\left[\alpha^{\prime}\right]^{\prime}}$. Then for $l \in L(k)$ :

$$
P_{[l \alpha]}=\nu_{l} P_{[\alpha]}=\nu_{l} H\left(P_{[1]}\right)=H \nu_{l} P_{[1]}=H P_{[l]}=H P_{[1]}=P_{[\alpha]} \text {. }
$$

That is $[\alpha]=[l \alpha]$. This implies that there exists an $l_{1} \in L(k)$ such that $\alpha=l \alpha l_{1}^{-1}$; that is $\alpha l_{1} \alpha^{-1} \in L(k)$. By [3,p. 187] $\alpha \in L(k)$; that is [ $\left.\alpha\right]=[1]$. Therefore $H\left(P_{[1]}\right)=P_{[1]}$. Similarly $H\left(P_{[1]^{\prime}}\right)=P_{[1]^{\prime}}$. Then

$$
H\left(P_{[\gamma]}\right)=H v_{\gamma} P_{[1]}=v_{\gamma} H\left(P_{[1]}\right)=\nu_{\gamma} P_{[1]}=P_{[\gamma]}
$$

and $H\left(P_{[\gamma]^{\prime}}\right)=P_{[\gamma]^{\prime}}, \forall \gamma \in L(k) * L\left(k^{\prime}\right)$. So $\left.H\right|_{P}=$ identity. Since $P$ is dense in $K,\left.H\right|_{K}=$ identity.

(b) Suppose $H\left(P_{[1]}\right)=P_{[\alpha]^{\prime}}$. A similar argument to part (a) establishes that this case never occurs.

Case 2. Suppose $H\left(P_{[1]}\right) \in D$. Notice by Proposition 2.2.1; if $l \in L(k)$ then $v_{l}\left(\bigcap_{i} D_{\gamma_{i}}\right) \neq\left(\bigcap_{i} D_{\gamma_{i}}\right)$. Then, if $H\left(P_{[1]}\right)=\bigcap_{i} D_{\gamma_{i}} ; v_{l}\left(\bigcap_{i} D_{\gamma_{i}}\right)=$ $v_{l} H\left(P_{[1]}\right)=H v_{l}\left(P_{[1]}\right)=H P_{[l]}=H P_{[1]}=\bigcap_{i} D_{\gamma_{i}}$. Contradiction. So $H\left(P_{[1]}\right)$ $\notin D$.

Since $H$ is periodic of period $p$, a prime, Smith theory implies that $F$, the fixed point set of $H$, is a generalized $Z_{p}$-cohomology manifold, with the 
$Z_{p}$-cohomology of a $k$-sphere, $k<4$.

Proposition 3.1.3 may be restated as $K \subset F$. That is, the fixed point set of $\widetilde{h}$ is $F-K$. Since $H$ and $L(k) * L\left(k^{\prime}\right)$ commute (Proposition 3.1.2), $F-K$ is invariant under the action of $L(k) * L\left(k^{\prime}\right)$, and hence $L(k) * L\left(k^{\prime}\right)$ acts freely on $F-K$. So

$$
\begin{array}{cl}
L(k) * L\left(k^{\prime}\right) \rightarrow F-K & \text { is a covering, where } \\
\downarrow & N \text { is the fixed point set } \\
N & \text { of } h .
\end{array}
$$

The spectral sequence of this covering has

$$
E_{s, t}^{2}=H_{s}\left(L(k) * L\left(k^{\prime}\right) ; H_{t}\left(F-K ; Z_{p}\right)\right) \Rightarrow H_{s+t}\left(N ; Z_{p}\right)
$$

where the homology of spaces is Čech homology and $H_{t}\left(F-K ; Z_{p}\right)$ is a module over $Z\left(L(k) * L\left(k^{\prime}\right)\right)$ in the obvious manner. Alexander duality implies $\widetilde{H}_{t}\left(F-K ; Z_{p}\right)=\widetilde{H}^{k-t-1}\left(K ; Z_{p}\right)$ where the tilde denotes reduced Čech homology or cohomology. By Corollary 2.2 .2 and since $K$ is zero dimensional

$$
\tilde{H}_{t}\left(F-K ; Z_{p}\right)=\left\{\begin{array}{l}
0 \quad \text { if } t \neq k-1, \\
Z\left(L * L^{\prime}\right) \otimes Z_{p} \text { if } t=k-1 .
\end{array}\right.
$$

LEMMA. $H_{r}\left(L(k) * L\left(k^{\prime}\right) ; H_{k-1}\left(F-K ; Z_{p}\right)\right)=0$ for $r \geqslant 1$.

PROoF. This follows easily from the fact that $H_{r}(\pi ; Z(\pi))=0$ for $r \geqslant 1$ where $Z(\pi)$ is a free $Z(\pi)$ module by multiplication on the left. For then the short exact sequence of $Z\left(L(k) * L\left(k^{\prime}\right)\right)$ modules

$$
0 \rightarrow Z\left(L(k) * L\left(k^{\prime}\right)\right) \stackrel{\mu}{\rightarrow} Z\left(L(k) * L\left(k^{\prime}\right)\right) \rightarrow Z_{p}\left(L(k) * L\left(k^{\prime}\right)\right) \rightarrow 0
$$

$(\mu$ is induced by multiplication by $p: Z \rightarrow Z$ ) yields a long exact sequence with low terms

$$
0 \rightarrow H_{1}\left(L(k) * L\left(k^{\prime}\right) ; H_{k-1}\left(F-K ; Z_{p}\right)\right) \rightarrow Z \stackrel{\times p}{\longrightarrow} Z \rightarrow \cdots
$$

so that $H_{1}\left(L(k) * L\left(k^{\prime}\right), H_{k-1}\left(F-K ; Z_{p}\right)\right)=0$. For higher values of $r$, the result is, of course, easier.

Assume $k=\operatorname{dim} F>1$. Then the only possible nonzero terms in the spectral sequence are $E_{s, t}^{2}$ where (a) $t=0$ or (b) $s=0$ and $t=k-1$. Since $L(k) * L\left(k^{\prime}\right)$ acts trivially on $H_{0}\left(F-K ; Z_{p}\right)=Z_{p}, E_{s, 0}^{2}=$ $H_{s}\left(L(k) * L\left(k^{\prime}\right) ; Z_{p}\right)$. In particular $E_{4,0}^{2} \neq 0$. The only possibly nonzero differential from $E_{4,0}^{2}$ is $d^{2}: E_{4,0}^{2} \rightarrow E_{4-k, k-1}^{2}$. But since $k<4,4-k \neq 0$, so $E_{4-k, k-1}^{2}=0$ and $d^{2}=0$. Therefore $0 \neq E_{4,0}^{2}=E_{4,0}^{\infty}=H_{4}\left(N ; Z_{p}\right)$. On the other hand, $N$, the fixed point set of $h$, is a generalized manifold of dimension $k<4$ so that $H_{4}\left(N ; Z_{p}\right)=0$. Contradiction. 
Assume, now, that $k=\operatorname{dim} F=1$ (notice: $K \subset F \Rightarrow \operatorname{dim} F \geqslant 1$ ). Then the only possibly nonzero terms at the $E^{2}$ level are $E_{s, 0}^{2}$. Again $E_{s, 0}^{2}=$ 0 for $s \geqslant 1$; in particular $E_{1,0}^{2}=0$. On the other hand, $H_{1}\left(N ; Z_{2}\right) \neq 0$, since $N$ is a (nonempty) bouquet of 1 -spheres. But this is a contradiction, since $E_{1,0}^{2}=E_{1,0}^{\infty}=H_{1}\left(N ; Z_{2}\right)$. Thus the assumption that $\operatorname{dim} F=k<4$ is incorrect. That is, $h$ is the identity.

REMARKS. 1. Glen Bredon has observed that a proof that no periodic map exists on $L$ can avoid any mention of the Freudenthal end point compactification. Indeed it is an elementary computation. I have felt justified in presenting this proof because the results and calculations of the ends seemed intrinsically interesting and also because it seemed to be in the spirit of the Smith approach to understanding periodic maps on covering spaces.

2. Conner and Raymond's example, $L(k)$, can easily be generalized to all higher dimensions, as can the construction and proof of the present paper. That is closed manifolds without any symmetry exist in all dimensions higher than three. I feel it would be interesting to construct a three dimensional example. A three dimensional example like those of Conner and Raymond would surely suffice.

3. All the examples known to me depend upon a result of Borel on aspherical spaces. This forces the present methods to yield examples with nontrivial fundamental group. Entirely different methods would be needed to construct simply connected manifolds.

\section{BIBLIOGRAPHY}

1. P. E. Conner and F. A. Raymond, Manifolds with few periodic homeomorphisms, Proceedings of the Second Conference on Compact Transformation Groups (University of Massachusetts, Amherst 1971), Lecture Notes in Math., vol. 299, Springer-Verlag, Berlin, 1972.

2. S. Mac Lane, Homology, Die Grundlehren der math. Wissenschaften, Band 114, Academic Press, New York; Springer-Verlag, Berlin, 1963. MR 28 \#122.

3. W. Magnus, A. Karrass and D. Solitar, Combinatorial group theory: Presentations of groups in terms of generators and relations, Pure and Appl. Math., vol. 13, Interscience, New York, 1966. MR 34 \#7617.

4. F. Raymond, The end point compactification of manifolds, Pacific J. Math. 10 (1960), 947-963. MR 22 \#11387.

5. D. Sullivan, unpublished.

DEPARTMENT OF MATHEMATICS, UNIVERSITY OF VIRGINIA, CHARLOTTESVILLE, VIRGINIA 22903 